Neurosurg Focus 33 (5):E9, 2012

\title{
On the value of routine prothrombin time screening in elective neurosurgical procedures
}

\author{
Stephan Dützmann, M.D., Florian Gerler, M.D., Gerhard Marquardt, M.D., Ph.D., \\ Volker Seifert, M.D., Ph.D., and Christian Senft, M.D., Ph.D.
}

Department of Neurosurgery, Johann Wolfgang Goethe University, Frankfurt/Main, Germany

\begin{abstract}
Object. The authors performed a study to evaluate whether preoperative assessment of prothrombin time (PT) is mandatory in patients undergoing routinely planned neurosurgical procedures.

Methods. The charts of all patients admitted to general wards of the authors' department for routinely planned surgery (excluding trauma and ICU patients) between 2006 and 2010 were retrospectively reviewed. The authors assessed preoperative PT and the clinical courses of all patients, with special consideration for patients receiving coagulation factor substitution. All cases involving hemorrhagic complications were analyzed in detail with regard to pre- and postoperative PT abnormalities. Prothrombin time was expressed as the international normalized ratio, and values greater than 1.28 were regarded as elevated.

Results. Clinical courses and PT values of 4310 patients were reviewed. Of these, 33 patients $(0.7 \%)$ suffered hemorrhagic complications requiring repeat surgery. Thirty-one patients $(94 \%)$ had a normal PT before the initial operation, while 2 patients had slightly elevated PT values of 1.33 and 1.65 , which were anticipated based on the patient's history. In the latter 2 cases, surgery was performed without prior correction of PT. Preoperatively, PT was elevated in 78 patients $(1.8 \%)$. In $73(93.6 \%)$ of the 78 patients, the PT elevation was expected and explained by each patient's medical history. In only $5(0.1 \%)$ of 4310 patients did we find an unexpected PT elevation (mean 1.53, range 1.37-1.74). All 5 patients underwent surgery without complications, while 2 had received coagulation factor substitution preoperatively, as requested by the surgeon, because of an estimated risk of bleeding complications. None of the 5 patients received coagulation factor substitution postoperatively, and later detailed laboratory studies ruled out single coagulation factor deficiencies. There was no statistically significant association between preoperatively elevated PT levels and the occurrence of hemorrhagic complications $(\mathrm{p}=0.12)$. Before the second procedure but not before the initial operation, $4(12 \%)$ of the 33 patients had elevated PT.

Conclusions. The findings suggest that the value of preoperative PT testing is limited in patients in whom a normal history can be ascertained. Close postoperative PT control is necessary in every neurosurgical patient, and better tests need to be developed to identify patients who are prone to hemorrhagic complications. (http://thejns.org/doi/abs/10.3171/2012.7.FOCUS12219)
\end{abstract}

\section{KEY WoRdS • prothrombin time • Coumadin • warfarin • screening}

$\mathrm{I}$ N most neurosurgical institutions it is common practice to assess coagulation parameters preoperatively in patients scheduled to undergo electively planned surgery because even small postoperative hemorrhages may have devastating effects. The standard screening tools are measurements of PT and aPTT. Although the value of these coagulation parameters for spontaneous intracranial hemorrhage is well established, ${ }^{23}$ their value for postoperative hemorrhage is unclear. Recently, PT and aPTT have been a topic of increased scrutiny and discussion. ${ }^{2,15}$

Prothrombin time values are assessed routinely, although their utility is unclear. ${ }^{22}$ Because almost all PT abnormalities that might complicate neurosurgical procedures can be derived from the patient's medical history, generalized testing may not be indispensable. Postopera-

\footnotetext{
Abbreviations used in this paper: aPTT = activated partial thromboplastin time; INR = international normalized ratio; $\mathrm{PT}=$ prothrombin time; PTT = partial thromboplastin time.
}

tive bleeding has been linked to multiple factors, ${ }^{7,11,17}$ but no study has convincingly proven that the dismissal of preoperative coagulation tests leads to more postoperative hemorrhages. An intact coagulation system is necessary for adequate hemostasis since multiple studies link (spontaneous) intracranial hemorrhage to a disturbed coagulation system. . $^{6,14,16,19,23,25,33}$

We hypothesized that preoperative assessment of the PT in patients undergoing routinely planned surgery is not indispensable but may be omitted in defined patient subgroups. Furthermore, if PT measurements at the initial presentation of a patient undergoing routinely planned surgery are of minor value in the prevention of postoperative hemorrhage, surgery-induced disturbance of the coagulation system may still play a role in patients with hemorrhagic complications. To elucidate the value of routine pre- and postoperative PT testing in neurosurgical patients, we retrospectively analyzed patient charts with regard to PT values and hemorrhagic complications. 


\section{S. Dützmann et al.}

\section{Methods}

The PT values of 4310 consecutive patients admitted to our neurosurgical service between 2006 and 2010 to undergo electively planned surgery were analyzed using the INR (developed in 1983 by Kirkwood ${ }^{20}$ ). Patients directly admitted to our ICU were excluded from this analysis because a complete medical history could not be obtained immediately. We selected patients with a PT greater than 1.28, which is considered "abnormal" at our institution. Furthermore we determined if elevated PT values could be explained and expected by patient history (for example, in patients receiving prior warfarin/Coumadin medication or in those with liver cirrhosis, and so on).

To elucidate the role of preoperative PT assessment in the prevention and/or prediction of postoperative hemorrhage, we retrospectively reviewed all patients with hemorrhagic complications that led to a reoperation with regard to pre- and postoperative PT abnormalities.

Institutional Policy for Preoperative Coagulation Testing and Factor Substitution

We screened every patient admitted to our service for coagulation factor abnormalities using laboratory testing of PT, aPTT, and fibrinogen. Using a questionnaire, we routinely obtained a specific history on bleeding problems. We checked levels of coagulation factor XIII in patients undergoing craniotomies because reduced levels have been shown to be associated with postoperative bleeding. ${ }^{20}$ If coagulation testing showed increased PT (INR > 1.28), patients were usually given prothrombin complex concentrate (PPSB-human, Octapharma) before surgery until PT normalized, and this was followed by further hemostaseological workup for specific coagulation factor deficits.

\section{Statistical Analysis}

Statistical analyses were performed using commercially available software (BIAS for Windows). Binomial dichotomized data were compared using the Fisher exact test, and categorical data were compared using the chisquare test. A p value $<0.05$ was considered statistically significant.

\section{Results}

\section{Preoperative PT Screening}

A total of 4310 patients were screened (Fig. 1). Of these, 170 patients $(3.9 \%)$ had a PT greater than 1.28 once during their hospital stay. Preoperatively, PT was elevated in 78 patients $(1.8 \%)$. In $73(93.6 \%)$ of the 78 patients the PT elevation was expected and explained by the patient's history (Table 1$)$. In only $5(0.1 \%)$ of 4310 patients did we find an unexpected PT elevation (mean 1.53, range 1.37-1.74) (Table 2).

Two of these 5 patients received coagulation factor substitution preoperatively by means of prothrombin complex concentrates in a dose-dependent fashion until PT normalized. These patients had only a slightly elevated INR, but the risk of postoperative bleeding in that specific surgery demanded substitution as requested by the surgeon. One patient had a vertebral body metastasis

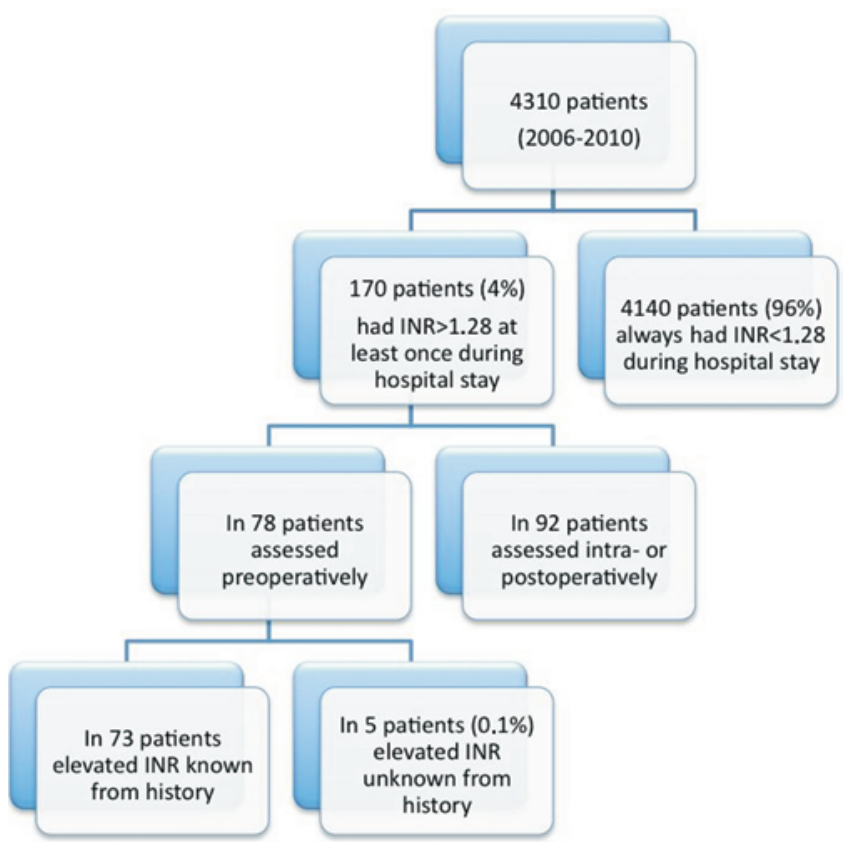

Fig. 1. Flowchart of 4310 patients who were admitted to our service between 2006 and 2010, excluding patients admitted to the ICU. We selected patients with an INR greater than 1.28 , which is considered elevated at our institution. Then we determined the timing of the PT measurement in reference to the operation (pre-, intra-, or postoperatively).

(preoperative INR 1.74) and the other patient had a spondylodiscitis (preoperative INR 1.55). Both patients underwent spinal instrumentation and stabilization procedures. Because none of these 5 patients had single coagulation factor deficits in later detailed laboratory studies, PT elevation was unexplained. It remained elevated in the patients in whom coagulation factor substitution was not administered throughout their hospital stay.

Postoperatively, none of the 5 patients with unexplained PT elevation received coagulation factor substitution. All surgeries and postoperative courses were uneventful.

\section{Retrospective Analysis of Hemorrhagic Complications}

In total, 33 patients $(0.77 \%)$ suffered bleeding compli-

TABLE 1: Summary of the known diseases or medications that caused coagulation disturbance in the 73 patients with preoperatively elevated $\mathrm{PT}^{*}$

\begin{tabular}{lc}
\hline Underlying Illness Causing Coagulation Disturbance & $\begin{array}{c}\text { No. of } \\
\text { Patients (\%) }\end{array}$ \\
\hline atrial fibrillation (warfarin medication) & $36(49)$ \\
aortic valve replacement (warfarin medication) & $14(19)$ \\
liver cirrhosis & $13(17)$ \\
status after deep vein thrombosis (warfarin medication) & $4(5)$ \\
status after lung embolism (warfarin medication) & $5(7)$ \\
renal artery stenosis (warfarin medication) & $1(1)$ \\
recovery phase after sepsis with DIC & $1(1)$ \\
\hline
\end{tabular}

* DIC = disseminated intravascular coagulation. 


\section{Prothrombin time screening}

TABLE 2: Overview of the 5 patients with elevated preoperative PT not explained by the patient's medical history or current medication*

\begin{tabular}{cclcccc}
\hline $\begin{array}{c}\text { Case } \\
\text { No. }\end{array}$ & $\begin{array}{c}\text { Age (yrs), } \\
\text { Sex }\end{array}$ & \multicolumn{1}{c}{ Disease } & $\begin{array}{c}\text { Possible } \\
\text { Underlying Disease }\end{array}$ & $\begin{array}{c}\text { Op } \\
\text { Delayed }\end{array}$ & $\begin{array}{c}\text { Coagulation Factor } \\
\text { Substitution (INR) }\end{array}$ & $\begin{array}{c}\text { Bleeding } \\
\text { Complications }\end{array}$ \\
\hline 1 & $75, \mathrm{~F}$ & vertebral body metastasis & prostate cancer & no & yes (1.74) & no \\
2 & $61, \mathrm{M}$ & vertebral body metastasis & prostate cancer & no & no (1.37) & no \\
3 & $78, \mathrm{~F}$ & spondylodiscitis & no & no & yes (1.55) & no \\
4 & $77, \mathrm{M}$ & spondylodiscitis & no & no & no (1.56) & no \\
5 & $61, \mathrm{~F}$ & herniated lumbar disc & no & no & no (1.47) & no \\
\hline
\end{tabular}

* Only 2 patients subsequently received coagulation factor substitution. In these patients, risk of postoperative bleeding in the specific surgery they underwent demanded substitution as requested by the surgeon. One patient (Case 1) had a vertebral body metastasis (preoperative INR 1.74) and the other patient (Case 3) had a spondylodiscitis (preoperative INR 1.55). Both had spinal instrumentation implanted. None of the 5 patients received factor substitution postoperatively. All surgeries were uneventful. Specific reason for the elevated INR was not found.

cations requiring revision surgery. Of these, $31(94 \%)$ had a normal PT before the initial operation, while 2 patients had elevated PT ( $\mathrm{p}=0.11$, Fisher exact test) (Table 3). These 2 patients had elevated PT preoperatively and were taken for emergency reoperation. One patient had intracranial metastastic disease of prostate cancer and underwent reoperation of a subdural hemorrhage after surgery the same day (initial preoperative PT 1.33). His slightly elevated PT was known prior to surgery and was attributed to warfarin medication that had been stopped and replaced with lowmolecular-weight heparin 5 days earlier. The other patient harbored a spinal metastatic lesion of a hepatocellular carcinoma and, 1 month after the initial surgery, underwent reoperation to treat a large hematoma in the subcutaneous and muscular tissue (initial preoperative PT 1.65); however, there were no signs of hemorrhagic complications in the early postoperative period. The patient's reduced liver function and low coagulation factor levels were also known prior to surgery. In both patients, surgeries were performed without prior correction of the PT level.

The mean PT values in patients who experienced complications was 1.08 (range 0.91-1.33) before and 1.23 (range 0.98-1.75) after the initial surgery, but before reoperation it was 1.23 (range 1.75-0.98). Four (12\%) of these 33 patients had elevated PT before the second surgery. One patient (postoperative PT 1.53) had spinal metastasis of a rhabdomyosarcoma and developed a disseminated intravascular coagulopathy. A large intraparenchymal hematoma developed in the subcutaneous and muscular tissue. Two patients had a glioblastoma (postoperative PTs

TABLE 3: Incidence of postoperative hemorrhage stratified by elevated versus normal preoperative $\mathrm{PT}^{*}$

\begin{tabular}{lccc}
\hline \multirow{2}{*}{ Variable } & \multicolumn{2}{c}{ Postop Hemorrhage } & \\
\cline { 2 - 3 } Yes & Notal No. of Cases \\
\hline elevated PT preop & 2 & 76 & 78 \\
normal PT preop & 31 & 4201 & 4232 \\
total & 33 & 4277 & 4310 \\
\hline
\end{tabular}

* Patients with elevated preoperative PT were not at a higher risk of hemorrhagic complications. See Results for full details.
1.37 and 1.35). One received intracranial adenovirus as part of a clinical trial; the other had a known factor XIII deficiency. Both patients developed postoperative epidural hematomas requiring evacuation. Finally, one patient had a giant olfactory groove meningioma and underwent a staged surgery (removal of the bulk of the tumor in a first step and subsequent completion of tumor removal after edema regression a few days later; postoperative PT 1.34). An epidural hematoma was evacuated on the same day as the first procedure.

The positive predictive value of a preoperatively elevated PT to indicate hemorrhagic complications in this population was $2.5 \%$. Its sensitivity and specificity were $6 \%$ and $98 \%$, respectively.

\section{Discussion}

Prothrombin time tests were developed to screen for specific coagulopathies in patients with a history of bleeding, and, thus, they were developed in cases with a high pretest probability. In contrast, the diseases for which screening is performed are rare, ${ }^{18}$ and, thus, the pretest probability and the yield are low. Still neurosurgeons worldwide routinely order PT testing preoperatively despite a lack of evidence for its usefulness. ${ }^{21}$

We show with this report that the value of unspecific preoperative PT testing is limited. Of 4310 patients scheduled for elective cases (thereby explicitly excluding trauma and ICU patients), less than $2 \%$ showed abnormal PT preoperatively altogether. We detected only 5 patients $(0.1 \%)$ with abnormal PTs that were unknown or unanticipated preoperatively from patient history. Those 5 patients had a mean PT of 1.53 (range 1.37-1.74), a value that was regarded safe by Matevosyan et al. ${ }^{22}$ None of these patients had single coagulation factor deficiencies in later detailed coagulation studies, which would have explained PT elevation. Thus, we could not find specific reasons or conditions that led to the elevated INR in these patients.

The evaluation of the PT as a measure of bleeding risk in patients undergoing invasive procedures has been investigated thoroughly. Some authors have argued that the relationship between PT and bleeding risk is remote at best. ${ }^{31}$ Even though evidence is growing that PT thresh- 


\section{S. Dützmann et al.}

olds to declare elective procedures to be safe should be moderately elevated, 3,32 the vast majority of studies claiming no increased bleeding risk in patients with high PTs undergoing invasive procedures have involved simple procedures such as placement of central intravenous lines or performance of thoracocentesis.,5,24,30 Similar data in neurosurgical procedures are lacking.

Prothrombin time elevation was not associated with postoperative hemorrhagic complications in the present series. We confirm the findings of Schramm and colleagues, ${ }^{29}$ who retrospectively examined 1211 neurosurgical cases and reported that abnormal PT was not predictive of postoperative bleeding. Thus, as was concluded by other nonneurosurgical reports, ${ }^{1,13}$ the utility of PT testing to detect unknown coagulopathies and to lower the bleeding complication rate should be reevaluated in elective neurosurgical procedures in which a normal patient history is obtained.

We found, however, that PT was elevated postoperatively in $12 \%$ of patients who suffered hemorrhagic complications before they were taken to the operating room for a rebleeding. This highlights the importance of tight postoperative PT control, because surgery is the main reason for depletion of coagulation factors. From the time that tissue has been damaged - that is, when surgery has started-the coagulation system is activated. Coagulation factors are consumed and levels decline. ${ }^{8}$ Depending on the extent, type, and duration of surgery, levels may decline to the extent that secondary coagulation is also impaired.

Therefore, better tests should be developed to broaden the existing testing armamentarium. ${ }^{10-12}$ These tests need to be tailored to the needs of the surgeon. In view of our results, they should be readily available intraoperatively to assess whether a formerly normal coagulation system gets disturbed through an operation. From an epidemiological point of view, hemorrhagic complications are caused by disturbances of thrombocyte function, which is not tested by measuring PT. ${ }^{28}$ Furthermore, preoperatively new concepts of hemostasis should be reflected. ${ }^{9}$ These tests should be able to mimic the complex interplay between activated thrombocytes and the coagulation system from tissue injury to forming a blood clot.

The main limitation of our study is its retrospective design. Generally, if we discovered an abnormal PT value, we chose to substitute coagulation factors. Therefore, one may not conclude from this study that PT testing should per se be abandoned for elective neurosurgical patients. To fully determine the value of PT testing and coagulation factor substitution, a randomized trial would be necessary; it is doubtful, however, whether an ethics committee would ever approve such a study. In a series of more than 4000 elective patients, however, we found only 5 with unexpectedly elevated PT.

From a cost perspective, one may argue that it not essential to refrain from PT testing: At our institution, laboratory costs for PT testing are low, approximately $\$ 7.50$ per test. The estimated annual amount needed to screen all elective patients preoperatively is around $\$ 8100$, which is arguably negligible given the overall department costs. On the other hand, none of the 5 patients with unexpected PT elevations experienced hemorrhagic complications, even if admittedly 2 of them received coagulation factors. Yet, all 5 patients' PT values were within the limits that Matevosyan et al. ${ }^{22}$ considered safe, so we may have exposed these patients to the potential risk of coagulation activation by administering prothrombin complex. ${ }^{27}$ Five patients are, however, too small a group to draw solid conclusions in this respect.

The positive predictive value of $2.5 \%$ is also limited by the fact that we provided coagulation factor substitution in most patients in whom we found elevated PTs, but again the necessity for substitution was anticipated based on patient history in the vast majority. The rate of unexpectedly elevated PTs that do not affect the bleeding tendency would be strongly diminished if testing were focused on elective patients with a higher pretest probability for PT abnormalities only-patients with current or previous anticoagulation medication, liver disease including alcoholism, or longer-lasting inflammatory or malignant illnesses. Subsequent studies might reveal specific conditions in which PT testing remains mandatory. Likewise, $\mathrm{Ng}$ et al. ${ }^{26}$ have suggested that the use of the preoperative coagulation test should be guided by clinical assessment rather than the procedure itself.

We conclude that it may be justified to refrain from routine PT testing in otherwise healthy individuals scheduled to undergo elective neurosurgical procedures who do not have a history of bleeding complications, known coagulopathies, liver disease (including alcoholism), or metastatic disease. If, however, PT testing reveals impaired coagulation capacity, thorough testing should nonetheless be performed to exclude single coagulation factor deficits, and PT should be corrected to reduce the risk of periprocedural hemorrhage.

\section{Conclusions}

The results of our study suggest that the yield of preoperative PT testing in routine elective cases in a neurosurgical population excluding trauma and ICU patients is trifling. Our data highlight the importance of surgery per se in initiating the coagulation cascade, consumption of coagulation factors, and bleeding complications in elective patients. Better tests need to be developed to broaden the existing testing armamentarium, aiming at identifying patients at risk for postoperative hemorrhagic complications.

\section{Disclosure}

The authors report no conflict of interest concerning the materials or methods used in this study or the findings specified in this paper.

Author contributions to the study and manuscript preparation include the following. Conception and design: Dützmann, Marquardt, Senft. Acquisition of data: Dützmann. Analysis and interpretation of data: Dützmann. Drafting the article: Dützmann. Critically revising the article: Geßler, Senft. Statistical analysis: Dützmann. Administrative/technical/material support: Seifert. Study supervision: Senft.

\section{Acknowledgment}

The authors thank Dr. Hans Ackermann for his statistical assistance 


\section{References}

1. Asaf T, Reuveni H, Yermiahu T, Leiberman A, Gurman G, Porat A, et al: The need for routine pre-operative coagulation screening tests (prothrombin time PT/partial thromboplastin time PTT) for healthy children undergoing elective tonsillectomy and/or adenoidectomy. Int J Pediatr Otorhinolaryngol 61:217-222, 2001

2. Burchiel KJ: Editorial. International normalized ratio. J Neurosurg 114:8, 2011

3. Davis JW, Davis IC, Bennink LD, Hysell SE, Curtis BV, Kaups KL, et al: Placement of intracranial pressure monitors: are "normal" coagulation parameters necessary? J Trauma 57:1173-1177, 2004

4. DeLoughery TG, Liebler JM, Simonds V, Goodnight SH: Invasive line placement in critically ill patients: do hemostatic defects matter? Transfusion 36:827-831, 1996

5. Fisher NC, Mutimer DJ: Central venous cannulation in patients with liver disease and coagulopathy-a prospective audit. Intensive Care Med 25:481-485, 1999

6. Forster MT, Mathé AK, Senft C, Scharrer I, Seifert V, Gerlach $\mathrm{R}$ : The influence of preoperative anticoagulation on outcome and quality of life after surgical treatment of chronic subdural hematoma. J Clin Neurosci 17:975-979, 2010

7. Fukamachi A, Koizumi H, Nukui H: Immediate postoperative seizures: incidence and computed tomographic findings. Surg Neurol 24:671-676, 1985

8. Gerald AG: Update on hemostasis: neurosurgery. Surgery 142 (4 Suppl):S55-S60, 2007

9. Gerlach R, Krause M, Seifert V, Goerlinger K: Hemostatic and hemorrhagic problems in neurosurgical patients. Acta Neurochir (Wien) 151:873-900, 2009

10. Gerlach R, Raabe A, Zimmermann M, Siegemund A, Seifert V: Factor XIII deficiency and postoperative hemorrhage after neurosurgical procedures. Surg Neurol 54:260-265, 2000

11. Gerlach R, Scheuer T, Beck J, Woszczyk A, Seifert V, Raabe A: Risk of postoperative hemorrhage after intracranial surgery after early nadroparin administration: results of a prospective study. Neurosurgery 53:1028-1035, 2003

12. Gerlach R, Tölle F, Raabe A, Zimmermann M, Siegemund A, Seifert V: Increased risk for postoperative hemorrhage after intracranial surgery in patients with decreased factor XIII activity: implications of a prospective study. Stroke 33:16181623,2002

13. Golub R, Cantu R, Sorrento JJ, Stein HD: Efficacy of preadmission testing in ambulatory surgical patients. Am J Surg 163:565-571, 1992

14. Gorter JW: Major bleeding during anticoagulation after cerebral ischemia: patterns and risk factors. Stroke Prevention In Reversible Ischemia Trial (SPIRIT). European Atrial Fibrillation Trial (EAFT) study groups. Neurology 53:1319-1327, 1999

15. Hoffer A, Selman W: Editorial. Transfusion. J Neurosurg 114: $1-2,2011$

16. Hylek EM, Singer DE: Risk factors for intracranial hemorrhage in outpatients taking warfarin. Ann Intern Med 120: 897-902, 1994

17. Kalfas IH, Little JR: Postoperative hemorrhage: a survey of 4992 intracranial procedures. Neurosurgery 23:343-347, 1988

18. Kamal AH, Tefferi A, Pruthi RK: How to interpret and pursue an abnormal prothrombin time, activated partial thromboplastin time, and bleeding time in adults. Mayo Clin Proc 82:864-873, 2007

19. Kawamata T, Takeshita M, Kubo O, Izawa M, Kagawa M, Takakura K: Management of intracranial hemorrhage associated with anticoagulant therapy. Surg Neurol 44:438-443, 1995
20. Kirkwood TB: Calibration of reference thromboplastins and standardisation of the prothrombin time ratio. Thromb Haemost 49:238-244, 1983

21. Kitchens CS: To bleed or not to bleed? Is that the question for the PTT? J Thromb Haemost 3:2607-2611, 2005

22. Matevosyan K, Madden C, Barnett SL, Beshay JE, Rutherford C, Sarode R: Coagulation factor levels in neurosurgical patients with mild prolongation of prothrombin time: effect on plasma transfusion therapy. Clinical article. J Neurosurg 114: 3-7, 2011

23. McCormick WF, Rosenfield DB: Massive brain hemorrhage: a review of 144 cases and an examination of their causes. Stroke 4:946-954, 1973

24. McVay PA, Toy PT: Lack of increased bleeding after paracentesis and thoracentesis in patients with mild coagulation abnormalities. Transfusion 31:164-171, 1991

25. Moskopp D, Brassel F, Ries F: [Intracranial and intraspinal hemorrhages in treatment with coumarin derivatives. Catamnesis of 63 cases between 1978 and 1986.] Klin Wochenschr 65:781-790, 1987 (Ger)

26. Ng KF, Lai KW, Tsang SF: Value of preoperative coagulation tests: reappraisal of major noncardiac surgery. World J Surg 26:515-520, 2002

27. Pabinger I, Brenner B, Kalina U, Knaub S, Nagy A, Ostermann $\mathrm{H}$, et al: Prothrombin complex concentrate (Beriplex P/N) for emergency anticoagulation reversal: a prospective multinational clinical trial. J Thromb Haemost 6:622-631, 2008

28. Pfanner G, Koscielny J, Pernerstorfer T, Gütl M, Perger P, Fries $\mathrm{D}$, et al: [Preoperative evaluation of the bleeding history. Recommendations of the working group on perioperative coagulation of the Austrian Society for Anaesthesia, Resuscitation and Intensive Care.] Anaesthesist 56:604-611, 2007 (Ger)

29. Schramm B, Leslie K, Myles PS, Hogan CJ: Coagulation studies in preoperative neurosurgical patients. Anaesth Intensive Care 29:388-392, 2001

30. Segal JB, Dzik WH: Paucity of studies to support that abnormal coagulation test results predict bleeding in the setting of invasive procedures: an evidence-based review. Transfusion 45:1413-1425, 2005

31. Tripodi A, Chantarangkul V, Primignani M, Fabris F, Dell'Era $A$, Sei C, et al: The international normalized ratio calibrated for cirrhosis (INR(liver)) normalizes prothrombin time results for model for end-stage liver disease calculation. Hepatology 46:520-527, 2007

32. West KL, Adamson C, Hoffman M: Prophylactic correction of the international normalized ratio in neurosurgery: a brief review of a brief literature. A review. J Neurosurg 114:9-18, 2011

33. Wijdicks EF, Schievink WI, Brown RD, Mullany CJ: The dilemma of discontinuation of anticoagulation therapy for patients with intracranial hemorrhage and mechanical heart valves. Neurosurgery 42:769-773, 1998

Manuscript submitted June 22, 2012.

Accepted July 19, 2012.

Part of this work was presented as an e-poster at the AANS Annual Meeting, Miami, Florida, April 14-18, 2012.

Please include this information when citing this paper: DOI: 10.3171/2012.7.FOCUS12219.

Address correspondence to: Stephan Dützmann, M.D., Department of Neurosurgery, Johann Wolfgang von Goethe University, Schleusenweg 2-18, D-60528 Frankfurt, Germany. email: Stephan. duetzmann@kgu.de. 